\title{
Peritoneal dialysis for autosomal dominant polycystic kidney disease: a retrospective study"
}

\author{
Xi-shao XIE, Zhou-tao XIE, Shi-long XIANG, Xing-qun YAN, \\ Xiao-hui ZHANG, Zhang-fei SHOU ${ }^{\dagger}$, Jiang-hua CHEN \\ (Kidney Disease Center, the First Affiliated Hospital, School of Medicine, Zhejiang University, Hangzhou 310003, China) \\ †E-mail: zfshou@zju.edu.cn \\ Received Sept. 26, 2015; Revision accepted Dec. 30, 2015; Crosschecked Apr. 15, 2016
}

\begin{abstract}
To describe the long-term clinical outcomes of patients with autosomal dominant polycystic kidney disease (ADPKD) who are on peritoneal dialysis (PD) therapy. We performed a retrospective matched-cohort analysis comparing the clinical outcomes of 30 ADPKD patients with those of 30 non-diabetic patients who had bilateral small kidneys between July 12007 and July 31 2014. The patient groups were matched by age, gender, and time of PD initiation. There were no significant differences in the demographic or biochemical parameters, comorbid conditions, residual glomerular filtration rate, or Charlson comorbidity score at the beginning of PD. The median renal volume was $1315 \mathrm{ml}$ for the ADPKD group and $213 \mathrm{ml}$ for the control group. Patients with ADPKD had similar 3-year patient survival (90.6\% versus $86.3 \%, P=0.807)$ and technique survival $(89.2 \%$ versus $74.3 \%, P=0.506)$ compared with non-ADPKD patients. Also, there was no significant difference in the peritonitis-free survival between the ADPKD and control groups $(P=0.22)$, and rates of peritonitis were similar ( 0.19 versus 0.21 episodes per patient-year, $P=0.26)$. No differences were observed in the incidence of PD-related complications, such as hernia and dialysate leak. ADPKD is not a contraindication for PD, and a subgroup of ADPKD patients with relatively small kidney volume can be treated using PD.
\end{abstract}

Key words: Peritoneal dialysis (PD), Autosomal dominant polycystic kidney disease (ADPKD), Outcome, Comorbidity http://dx.doi.org/10.1631/jzus.B1500235

CLC number: R459.5

\section{Introduction}

Autosomal dominant polycystic kidney disease (ADPKD), the most common inherited kidney disease, which affects more than 12.5 million individuals worldwide (Chapman et al., 2015), is the fourth most common cause of end-stage renal disease (ESRD) in both China and the western world. ADPKD is responsible for $3 \%-10 \%$ of all patients treated for renal replacement therapy (Liu, 2013; Spithoven et al., 2014). Transplantation is the optimal renal replacement therapy in appropriate patients with ADPKD. When transplantation is not an option, or for those

\footnotetext{
¿ Corresponding author

* Project supported by the National Natural Science Foundation of China (Nos. 81170707 and 81300619)

(1) ORCID: Xi-shao XIE, http://orcid.org/0000-0001-9049-1701

(C) Zhejiang University and Springer-Verlag Berlin Heidelberg 2016
}

awaiting transplantation, peritoneal dialysis (PD) may be an option. However, PD has not been considered a suitable method for ADPKD patients by many nephrologists because the progressive development of kidney cysts might reduce the intraperitoneal space and increase intraperitoneal pressure, resulting in pressure-driven complications and poor dialysis efficacy. del Peso et al. (2003) and Fletcher et al. (1994) reported that ADPKD patients on PD therapy may experience more abdominal wall hernias and peritoneal leaks than patients with a different type of nephropathy. However, in recent years, some studies from the UK, France, Spain, and Hong Kong Special Administrative Region (China) reported that PD in ADPKD patients has similar patient and technical survivals compared with non-ADPKD patients $(\mathrm{Ku}-$ mar et al., 2008; Li et al., 2011; Lobbedez et al., 2011; Janeiro et al., 2015). 
Under the guidance of government and support policies, $\mathrm{PD}$ has been rapidly developed in China in recent years; approximately 46000 of patients with ESRD received PD in the mainland China at the end of 2013 (Yu and Yang, 2015). An increased number of patients, including some ADPKD patients, chose $\mathrm{PD}$ as their primary renal replacement therapy. Thus, we performed a retrospective study to describe the long-term outcomes of PD in ADPKD patients compared with non-ADPKD patients at our centre.

\section{Materials and methods}

\subsection{Patients}

We reviewed all incident ADPKD patients starting PD at our centre between July 12007 and July 31 2014. For each ADPKD patient, one new PD patient who had the same gender and a similar age, had a non-diabetic cause of ESRD with bilateral small kidneys, and had started PD immediately before or after the ADPKD patient, was selected to be in the control group. We excluded patients who were younger than 18 years of age, were previously treated with haemodialysis for more than 3 months, or had undergone kidney transplantation.

\subsection{Data collection}

Clinical data, including demographic parameters, biochemical data, comorbidity, details of peritonitis, and transplantation, were collected from our regularly updated computerized records based on a review of patient medical and nursing notes. Demographic data were collected at the beginning of PD, including the gender, age, body mass index (BMI), cause of ESRD, and comorbid conditions (history of angina, myocardial infarction, cerebrovascular disease, chronic pulmonary disease, diabetes (not as the cause of ESRD), peripheral vascular disease, and malignancy). The presence of each disease was scored, and the Charlson comorbidity index (CCI) score was calculated (Beddhu et al., 2000). The renal volume was defined as the total volume of both kidneys and calculated based on ultrasound measurements (FickBrosnahan et al., 2002). The collected biochemical data included the baseline, haemoglobin, serum albumin, uric acid, phosphates, calcium, and cholesterol levels. Peritoneal membrane transport and dialysate- to-plasma ratios of creatinine (D:Pcr) at $4 \mathrm{~h}$ were assessed using the standard peritoneal equilibration test (Twardowski et al., 1987). The dialysis dose $(\mathrm{Kt} / \mathrm{V})$ was calculated from the 24-h dialysate collection using PD Adequest software (Baxter Healthcare Corp., Deerfield, IL, USA). Residual renal function was calculated as the mean of urea and creatinine clearance, and it was adjusted for $1.73 \mathrm{~m}^{2}$ body surface area from a 24-h urine collection. Data related to the extra-renal complications of ADPKD were specifically collected, including hernia, dialysate leaks, nephrectomy, bowel perforation, diverticular disease, and intracranial haemorrhage.

\subsection{Clinical outcomes}

The diagnosis of peritonitis was based on the International Society for Peritoneal Dialysis (ISPD) guidelines (Li et al., 2010) with at least 2 of the following criteria: abdominal pain or cloudy PD effluent, leucocytosis in peritoneal fluid effluent (white blood cell count $>100 \mu \mathrm{l}^{-1}$ ), or positive Gram stain or bacterial culture from effluent. Death within $30 \mathrm{~d}$ of switching to haemodialysis therapy was considered death on PD therapy, with the assumption that deaths occurring during this period were likely to be associated with PD. Permanent cessation of PD therapy, due to PD-relative complications, was defined as a technique failure. All patients were followed up until death, kidney transplantation, transfer to haemodialysis therapy, recovery of kidney function, loss to follow-up, transfer to another dialysis centre, or censoring on July 31, 2014.

\subsection{Statistical analysis}

Continuous variables are presented as the mean and standard deviation or median with interquartile range, and categorical variables are presented as the frequencies with percentages. Differences between groups were analyzed by the $t$-test for normally distributed continuous data, the Mann-Whitney $U$-test for skewed continuous data, and chi-squared test for categorical data. The patient, technique, and peritonitisfree survivals were assessed by the Kaplan-Meier method until an event or July 312014 (any other outcome was considered censored data), and groups were compared using the log-rank test. Because of the limited sample size and small number of events, the Cox regression model was not performed for survival 
analysis. All statistical analyses were performed using SPSS software Version 19.0 (SPSS Inc., http:// www.spss.com). A $P$-value less than 0.05 was considered statistically significant.

\section{Results}

\subsection{Patients' characteristics}

A total of 30 patients with ADPKD who started PD in our centre from July 12007 to July 312014 were included, and they were compared with 30 matched controls. Baseline demographic and clinic characteristics are shown in Table 1. There were no significant differences in the demographic or biochemical parameters, comorbid conditions, or Charlson comorbidity score. Residual glomerular filtration rate (GFR) at the beginning of PD in the ADPKD group was similar to that in the control group. The mean renal volume was $1315 \mathrm{ml}$ for the ADPKD group and $213 \mathrm{ml}$ for the control group. No significant differences were noted in the $\mathrm{Kt} / \mathrm{V}$ or D:Pcr between the two groups.

Table 1 Baseline demographics and clinical characteristics of ADPKD and control patients

\begin{tabular}{|c|c|c|c|}
\hline Characteristics & $\operatorname{ADPKD}(n=30)$ & Control $(n=30)$ & $P$ \\
\hline Age (year) & $52.5 \pm 11.0$ & $52.6 \pm 11.1$ & 0.962 \\
\hline Male (\%) & $18(60 \%)$ & $18(60 \%)$ & 1.000 \\
\hline BMI & $22.1 \pm 2.6$ & $21.7 \pm 3.0$ & 0.587 \\
\hline \multicolumn{4}{|l|}{ Blood pressure } \\
\hline Systolic & $133(120-148)$ & $141(124-154)$ & 0.041 \\
\hline Diastolic & $84(75-92)$ & $83(75-92)$ & 0.332 \\
\hline \multicolumn{4}{|l|}{ Comorbid conditions } \\
\hline Hypertension & $30(100 \%)$ & $28(93.3 \%)$ & 0.152 \\
\hline Diabetes & $1(3.3 \%)$ & $0(0 \%)$ & 0.313 \\
\hline Heart disease & $5(16.7 \%)$ & $4(13.3 \%)$ & 0.718 \\
\hline Cerebrovascular & $1(3.3 \%)$ & $2(6.7 \%)$ & 0.553 \\
\hline Charlson index score & $3.3 \pm 1.1$ & $3.1 \pm 1.0$ & 0.351 \\
\hline \multicolumn{4}{|l|}{ Baseline biochemistry } \\
\hline Haemoglobin $(\mathrm{g} / \mathrm{dl})$ & $8.1 \pm 1.6$ & $8.0 \pm 1.5$ & 0.957 \\
\hline Serum albumin $(\mathrm{g} / \mathrm{dl})$ & $4.1 \pm 5.2$ & $3.9 \pm 6.4$ & 0.182 \\
\hline Serum uric acid (mg/dl) & $8.1 \pm 2.5$ & $8.5 \pm 2.0$ & 0.131 \\
\hline Calcium (mg/dl) & $8.2 \pm 1.2$ & $8.4 \pm 1.0$ & 0.529 \\
\hline Phosphate (mg/dl) & $5.5 \pm 1.3$ & $5.7 \pm 1.5$ & 0.630 \\
\hline Triglycerides (mg/dl) & $137.2 \pm 88.5$ & $141.6 \pm 75.2$ & 0.662 \\
\hline Total cholesterol (mg/dl) & $140.8 \pm 36.9$ & $167.3 \pm 75.4$ & 0.213 \\
\hline HDL-C (mg/dl) & $32.7 \pm 8.5$ & $40.4 \pm 14.3$ & 0.016 \\
\hline LDL-C (mg/dl) & $76.2 \pm 26.9$ & $91.2 \pm 48.8$ & 0.150 \\
\hline Residual GFR (ml/min per $1.73 \mathrm{~m}^{2}$ ) & $7.4 \pm 2.6$ & $6.9 \pm 3.1$ & 0.458 \\
\hline 24-h urine output & $1100(800-1500)$ & $1000(765-1400)$ & 0.253 \\
\hline Renal volume (ml) & $1315(1240-1426)$ & $213(197-226)$ & $<0.001$ \\
\hline Total Kt/V & $2.11 \pm 0.53$ & $2.08 \pm 0.56$ & 0.328 \\
\hline Transporter status & & & 0.180 \\
\hline High average and high & $8(26.7 \%)$ & $13(43.3 \%)$ & \\
\hline Low average and low & $22(73.3 \%)$ & $17(56.7 \%)$ & \\
\hline D:Pcr (4 h) & $0.61(0.58-0.66)$ & $0.63(0.57-0.75)$ & 0.463 \\
\hline
\end{tabular}

ADPKD: autosomal dominant polycystic kidney disease; BMI: body mass index; HDL-C: high-density lipoprotein cholesterol; LDL-C: low-density lipoprotein cholesterol; GFR: glomerular filtration rate; D:Pcr $(4 \mathrm{~h})$ : dialysate-to-plasma ratios of creatinine at $4 \mathrm{~h}$. Continuous variables are presented as the mean and standard deviation or median with interquartile range, and categorical variables are presented as the frequencies with percentages 


\subsection{Patient and technique survivals}

The average duration of follow-up was 27.1 months for the ADPKD group and 27.0 months for the control group $(P=0.961)$. The patient outcomes are shown in Table 2. Three patients died in the ADPKD group (one due to stroke, one due to malnutrition, and one due to cardiovascular accident) and two died in the control group (one due to severe infection and one due to unknown reasons). Five ADPKD patients and six patients in the control group were transferred to hemodialysis (HD) permanently because of technique failure. At the 3rd year, the patient survival was $90.6 \%$ and $86.3 \%$ for the ADPKD and control groups, respectively (log-rank test, $P=0.807$; Fig. 1 ), whereas the technique survival rates were $89.2 \%$ and $74.3 \%$, respectively (log-rank test, $P=0.506$; Fig. 2).

Table 2 Clinical outcomes of ADPKD and control patients

\begin{tabular}{lccc}
\hline \multicolumn{1}{c}{ Outcome } & $\begin{array}{c}\text { ADPKD } \\
(n=30)\end{array}$ & $\begin{array}{c}\text { Control } \\
(n=30)\end{array}$ & $P$ \\
\hline Death & $3(10 \%)$ & $2(6.7 \%)$ & 0.807 \\
Conversion to & $5(16.7 \%)$ & $6(20 \%)$ & 0.506 \\
$\quad$ haemodialysis & & & \\
Peritonitis & 2 & 1 & \\
Membrane failure & 0 & 2 & \\
Leak & 1 & 0 & \\
Hernia & 2 & 1 & \\
Others & 1 & 2 & \\
Transplant & 5 & 5 & 1.000 \\
Loss to follow-up & 0 & 0 & \\
\hline
\end{tabular}

ADPKD: autosomal dominant polycystic kidney disease

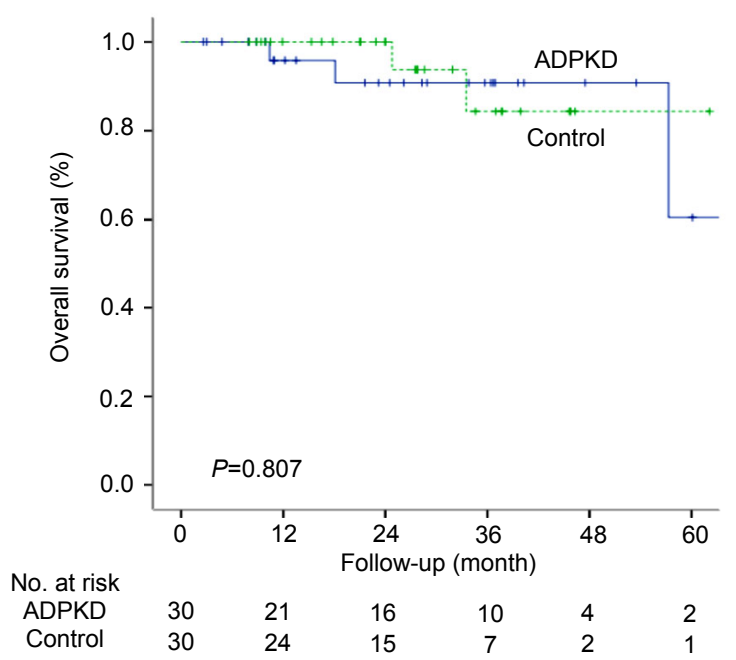

Fig. 1 Kaplan-Meier estimates of the patient survival in ADPKD and control patients (log-rank test, $P=0.807$ )

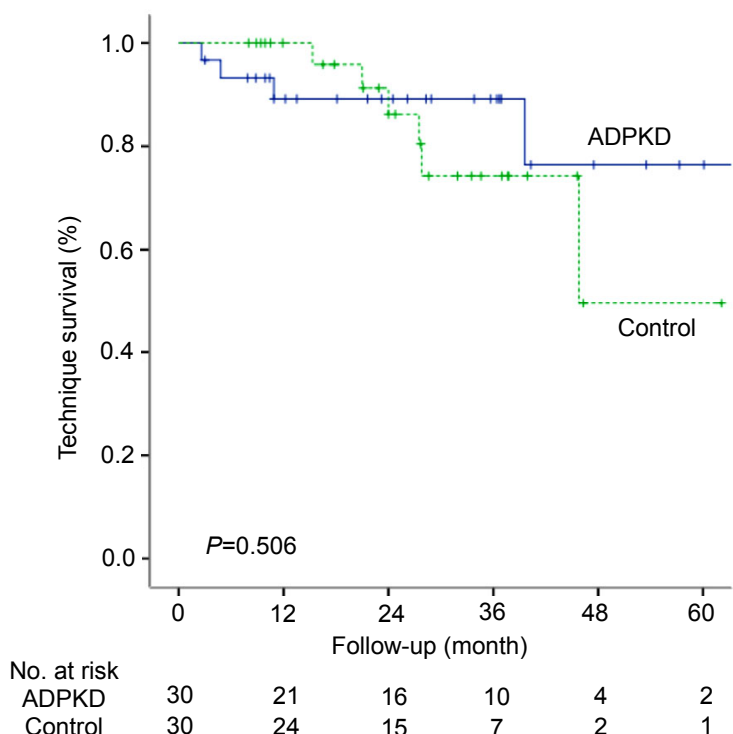

Fig. 2 Kaplan-Meier estimates of technique survival in ADPKD and control patients (log-rank test, $P=0.506$ )

\subsection{Peritonitis}

During the study period, $11(36.7 \%)$ patients presented with a total of 13 episodes of peritonitis in the ADPKD group, whereas 14 peritonitis episodes were diagnosed in $7(23.3 \%)$ patients in the control group $(P=0.260)$. The peritonitis rates were 0.19 and 0.21 episodes per patient-year for the ADPKD and control groups, respectively. The number of peritonitis cases requiring catheter removal was 2 in the ADPKD group and 1 in the control group. No significant difference was noted in the distribution of causative organisms between two groups (Table 3). There was also no significant difference in the peritonitis-free survival between groups (log-rank test, $P=0.218$; Fig. 3).

\subsection{Other complications}

None of the patients had abdominal wall hernias before the initiation of PD therapy. During the period of PD, 4 patients in the ADPKD group and 2 in the control group developed hernias (Table 4). Two patients in the ADPKD group (one due to recurrent hernia and the other due to patient's choice) and one in the control group (due to patient's choice) permanently transferred to $\mathrm{HD}$, while others were able to resume $\mathrm{PD}$ after surgical repair. One patient in the ADPKD group had a dialysate leak (hydrocele of tunica vaginalis) and required conversion to HD. One patient with ADPKD underwent nephrectomy and then transferred to HD. 


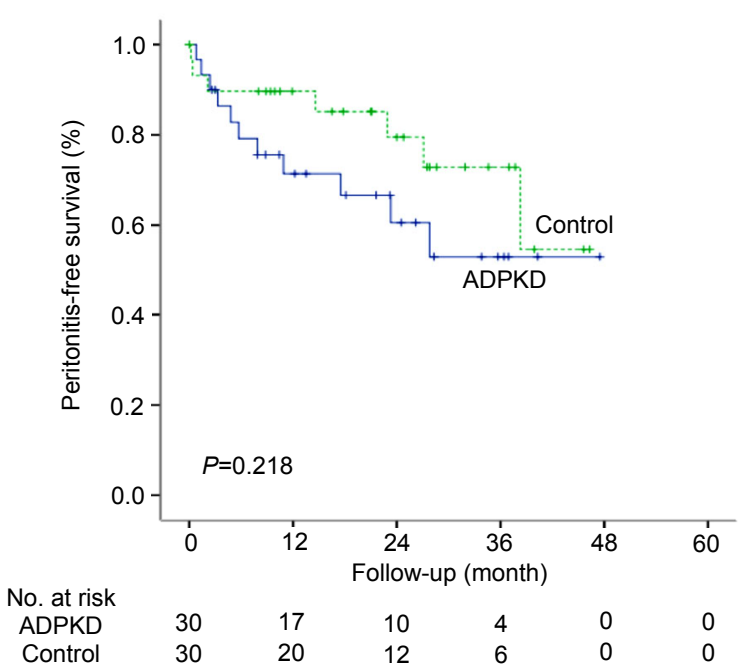

Fig. 3 Kaplan-Meier estimates of peritonitis-free survival in ADPKD and control patients (log-rank test, $P=0.218$ )

Table 3 Peritonitis during PD therapy

\begin{tabular}{lccc}
\hline \multicolumn{1}{c}{ Characteristics } & $\begin{array}{c}\text { ADPKD } \\
(n=30)\end{array}$ & $\begin{array}{c}\text { Control } \\
(n=30)\end{array}$ & $P$ \\
\hline No. of patients with & 11 & 7 & 0.260 \\
peritonitis & $(36.7 \%)$ & $(23.3 \%)$ & \\
Total No. of episodes & 13 & 14 & \\
Gram-negative & 3 & 3 & 1.000 \\
Escherichia coli & 3 & 1 & \\
Others & 0 & 2 & \\
Gram-positive & 8 & 5 & 0.180 \\
CNSS & 6 & 2 & \\
Staphylococcus aureus & 0 & 1 & \\
Streptococcus species & 1 & 0 & \\
Enterococcus species & 1 & 1 & \\
Others & 0 & 1 & \\
Fungus & 1 & 1 & \\
Culture-negative & 1 & 5 & \\
Episodes requiring & 2 & 1 & \\
catheter removal & & & \\
\hline ADPKD: & & &
\end{tabular}

ADPKD: autosomal dominant polycystic kidney disease; CNSS: coagulase negative staphylococcal species

Table 4 Complications during follow-up (not including peritonitis)

\begin{tabular}{lcc}
\hline \multicolumn{1}{c}{ Complication } & $\begin{array}{c}\text { ADPKD } \\
(n=30)\end{array}$ & $\begin{array}{c}\text { Control } \\
(n=30)\end{array}$ \\
\hline Leak & 1 & 0 \\
Hernia & 4 & 2 \\
Nephrectomy & 1 & 0 \\
Cardiovascular accident & 3 & 1 \\
Cerebrovascular accident & 0 & 2 \\
Non-peritonitis infection & 3 & 2 \\
\hline
\end{tabular}

ADPKD: autosomal dominant polycystic kidney disease

\section{Discussion}

In this retrospective study, we included all patients with ADPKD who chose PD as their first-line renal replacement therapy in our hospital between July 12007 and July 31 2014. The patient and technique survivals of ADPKD patients on PD were similar to those for non-ADPKD patients. No significant differences in the overall incidence of peritonitis or other complications were observed between the two groups.

Traditionally, ADPKD patients were thought to be poorer candidates for PD therapy than patients with other types of nephropathy. In some earlier reports, it was believed that enlarged kidneys and livers would reduce the intraperitoneal space and increase intraperitoneal pressure. del Peso et al. (2003) and Fletcher et al. (1994) reported that the ADPKD patients on PD therapy had a significantly increased risk for developing abdominal wall hernias and acute hydrothorax than patients with a different type of nephropathy, and the efficacy of PD therapy would be impaired by the reduced intraperitoneal space and effective peritoneal surface area. As a result, PD therapy has not been considered to be a suitable method for ADPKD patients by many nephrologists. However, in recent years, different results have been reported by several studies. Kumar et al. (2008) observed a cohort of 56 ADPKD patients who were started on PD therapy over a 12-year period and found that the long-term outcomes (mortality and technique survival) were similar to those of a matched control group of non-diabetic patients with bilateral small kidneys. Li et al. (2011) and Janeiro et al. (2015) also found similar outcomes between ADPKD patients and controls. In our retrospective study, no significant differences were observed in the patient or technique survival between ADPKD patients and matched controls.

del Peso et al. (2003) reported that the ADPKD patients on PD therapy had a four-fold higher risk for developing abdominal wall hernias than patients with a different type of nephropathy, which could result in technique failure. However, Kumar et al. (2008) and Li et al. (2011) reported that most hernia patients could resume PD therapy after surgical repair. In our study, the hernia incidence was similar between the two groups (4 in the ADPKD group and 3 in the 
control group). Two ADPKD patients permanently transferred to $\mathrm{HD}$, one because of recurrent hernia after surgical repair and the other because of the patient's choice. Some studies reported that dialysate leak was more common in patients with ADPKD (Fletcher et al., 1994; Hadimeri et al., 1998), but only one ADPKD patient in our study developed dialysate leak and transferred to HD. Our results were similar to the findings by Li et al. (2011) and Janeiro et al. (2015).

Although ADPKD patients have an increased prevalence of colonic diverticula, and are susceptible to Gram-negative peritonitis (that is not increased), we found no difference in the risk of developing peritonitis between ADPKD and other non-diabetic PD patients. The distribution of causative organisms was also similar between the two groups. Our result is in agreement with several previous reports (Pandya et al., 2004; Kumar et al., 2008; Li et al., 2011).

Unavoidably, there are several limitations to this study. First, this was a single-centre observational study with a small sample size. Second, the renal volume was calculated using ultrasonography, which was operator-dependent and less accurate than computed tomography (CT) or magnetic resonance imaging (MRI) (Grantham et al., 2006; Bae and Grantham, 2010). For most ADPKD patients who received PD therapy in our unit, the kidney volume was relatively small; if the inferior pole of the kidney was below the level of umbilicus, PD therapy would not be offered. We did not measure the rates of change in the kidney volume during follow-up; as a result, the relationship between the kidney volume growth and clinical outcomes could not be assessed in our study.

\section{Conclusions}

Our result demonstrates that the patient and technique survivals of ADPKD patients on PD therapy are similar to those of the non-diabetic patients with bilateral small kidneys. ADPKD is not a contraindication to PD. A subgroup of ADPKD patients with a relatively small kidney volume can be treated using PD.

\section{Acknowledgements}

We thank Duo LYU from Institute of Clinical Trial, the First Affiliated Hospital, School of Medicine, Zhejiang University, China for her assistance in statistical analysis.

\section{Compliance with ethics guidelines}

Xi-shao XIE, Zhou-tao XIE, Shi-long XIANG, Xing-qun YAN, Xiao-hui ZHANG, Zhang-fei SHOU, and Jiang-hua CHEN declare that they have no conflict of interest.

All procedures followed were in accordance with the ethical standards of the responsible committee on human experimentation (institutional and national) and with the Helsinki Declaration of 1975, as revised in 2008 (5). Informed consent was obtained from all patients for being included in the study. Additional informed consent was obtained from all patients for whom identifying information is included in this article.

\section{References}

Bae, K.T., Grantham, J.J., 2010. Imaging for the prognosis of autosomal dominant polycystic kidney disease. Nat. Rev. Nephrol., 6(2):96-106. http://dx.doi.org/10.1038/nrneph.2009.214

Beddhu, S., Bruns, F.J., Saul, M., et al., 2000. A simple comorbidity scale predicts clinical outcomes and costs in dialysis patients. Am. J. Med., 108(8):609-613. http://dx.doi.org/10.1016/S0002-9343(00)00371-5

Chapman, A.B., Devuyst, O., Eckardt, K.U., et al., 2015. Autosomal-dominant polycystic kidney disease (ADPKD): executive summary from a Kidney Disease: Improving Global Outcomes (KDIGO) Controversies Conference. Kidney Int., 88(1):17-27. http://dx.doi.org/10.1038/ki.2015.59

del Peso, G., Bajo, M.A., Costero, O., et al., 2003. Risk factors for abdominal wall complications in peritoneal dialysis patients. Perit. Dial. Int., 23(3):249-254.

Fick-Brosnahan, G.M., Belz, M.M., McFann, K.K., et al., 2002. Relationship between renal volume growth and renal function in autosomal dominant polycystic kidney disease: a longitudinal study. Am. J. Kidney Dis., 39(6): $1127-1134$. http://dx.doi.org/10.1053/ajkd.2002.33379

Fletcher, S., Turney, J.H., Brownjohn, A.M., 1994. Increased incidence of hydrothorax complicating peritoneal dialysis in patients with adult polycystic kidney disease. Nephrol. Dial. Transplant., 9(7):832-833.

Grantham, J.J., Torres, V.E., Chapman, A.B., et al., 2006. Volume progression in polycystic kidney disease. N. Engl. J. Med., 354(20):2122-2130. http://dx.doi.org/10.1056/NEJMoa054341

Hadimeri, H., Johansson, A.C., Haraldsson, B., et al., 1998. CAPD in patients with autosomal dominant polycystic kidney disease. Perit. Dial. Int., 18(4):429-432.

Janeiro, D., Portoles, J., Maria Tato, A., et al., 2015. Peritoneal dialysis can be an option for dominant polycystic kidney disease: an observational study. Perit. Dial. Int., 35(5): 530-536. http://dx.doi.org/10.3747/pdi.2014.00029

Kumar, S., Fan, S.L., Raftery, M.J., et al., 2008. Long term outcome of patients with autosomal dominant polycystic kidney diseases receiving peritoneal dialysis. Kidney Int., 
74(7):946-951.

http://dx.doi.org/10.1038/ki.2008.352

Li, L., Szeto, C.C., Kwan, B.C., et al., 2011. Peritoneal dialysis as the first-line renal replacement therapy in patients with autosomal dominant polycystic kidney disease. Am. J. Kidney Dis., 57(6):903-907. http://dx.doi.org/10.1053/j.ajkd.2011.01.019

Li, P.K., Szeto, C.C., Piraino, B., et al., 2010. Peritoneal dialysis-related infections recommendations: 2010 update. Perit. Dial. Int., 30(4):393-423. http://dx.doi.org/10.3747/pdi.2010.00049

Liu, Z.H., 2013. Nephrology in China. Nat. Rev. Nephrol., 9(9): 523-528. http://dx.doi.org/10.1038/nrneph.2013.146

Lobbedez, T., Touam, M., Evans, D., et al., 2011. Peritoneal dialysis in polycystic kidney disease patients. Report from the French peritoneal dialysis registry (RDPLF). Nephrol. Dial. Transplant., 26(7):2332-2339. http://dx.doi.org/10.1093/ndt/gfq712

Pandya, B.K., Friede, T., Williams, J.D., 2004. A comparison of peritonitis in polycystic and non-polycystic patients on peritoneal dialysis. Perit. Dial. Int., 24(1):79-81.

Spithoven, E.M., Kramer, A., Meijer, E., et al., 2014. Renal replacement therapy for autosomal dominant polycystic kidney disease (ADPKD) in Europe: prevalence and survival - an analysis of data from the ERA-EDTA Registry. Nephrol. Dial. Transplant., 29(Suppl. 4):iv15-iv25. http://dx.doi.org/10.1093/ndt/gfu017

Twardowski, Z.J., Nolph, K.D., Khanna, R., et al., 1987. Peritoneal equilibration test. Perit. Dial. Int., 7(3):138-147.

Yu, X., Yang, X., 2015. Peritoneal dialysis in China: meeting the challenge of chronic kidney failure. Am. J. Kidney Dis., 65(1):147-151.

http://dx.doi.org/10.1053/j.ajkd.2014.08.023

\section{中文概要}

题 目: 多囊肾患者行腹膜透析的预后分析

目 的: 探讨多囊肾致终末期肾病患者行腹膜透析治疗的 预后情况。

创新点: 分析国内最大一组单中心样本关于多囊肾患者行 腹膜透析治疗的预后研究, 进一步证实多囊肾并 非腹膜透析的禁忌症。

方 法: 回顾性分析 2007 年 7 月 1 日至 2014 年 7 月 31 日于我院置管并开始维持性腹膜透析的 30 例多 囊肾致终末期肾病患者, 选择 30 例年龄及性别 与多囊肾组匹配的非多囊肾患者作为对照组。记 录患者一般资料、透析初始资料、并发症、生存 时间、退出透析或死亡等治疗结局。采用 Kaplan-Meier 法和 log-rank 检验进行生存分析。

结 论: 多囊肾组 3 年病人生存率和技术生存率分别为 $90.6 \%$ 和 $89.2 \%$, 对照组为 $86.3 \%$ 和 $74.3 \%$, 差异 无统计学意义（图 1 和 2)。两组患者的腹膜炎 发生率分别为 0.19 次/病人年和 0.21 次/病人年 （表 3）。两组患者腹透液渗漏、疝气、心脑血 管事件发生率之间的差异均无统计学意义 (表 4)。 综上所述, 通过合理的病人篮选和评估, 多囊肾 不是腹膜透析的禁忌症。

关键词: 腹膜透析; 多囊肾; 预后; 并发症 